INPLASY

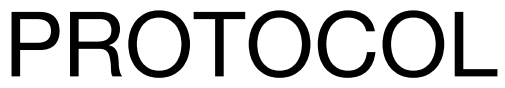

To cite: Xiao et al. Efficacy and safety of acupuncture in the treatment of postprandial distress syndrome: A systematic review and metaanalysis. Inplasy protocol

2021120101. doi:

10.37766/inplasy2021.12.0101

Received: 23 December 2021

Published: 23 December 2021

Corresponding author:

Fangli Xiong

xfl580321@126.com

Author Affiliation:

First Affiliated Hospital of Guizhou University of Traditional Chinese Medicine, Guizhou University of Traditional Chinese Medicine, Guiyang, Guizhou, China.

Support: No financial support.

Review Stage at time of this submission: The review has not yet started.

Conflicts of interest:

None declared.

\section{Efficacy and safety of acupuncture in the treatment of postprandial distress syndrome: A systematic review and meta-analysis}

Xiao, G'; Zhao, Y2; Chen, $X^{3}$.

Review question / Objective: Traditional Chinese medicine advocates using acupuncture for the treatment of postprandial distress syndrome (PDS) in people with functional dyspepsia (FD), but large clinical trials of acupuncture have produced controversial results. We perform this meta-analysis to evaluate the efficacy and safety of acupuncture in the treatment of postprandial distress syndrome. P: The subjects were patients diagnosed with postprandial discomfort syndrome. I: Patients received acupuncture as a treatment. C: Subjects received acupuncture in non-acupoint points, Gasmotin, moroxyline, or other reported interventions. O: The main outcomes included in this meta-analysis are the overall therapeutic rate, the Symptom Index of Dyspepsia (SID), the Hospital Anxiety Depression Scale (HADS), the Nepean Dyspepsia Index (NDI), and the incidence of the side effects.

Condition being studied: Efficacy and safety of acupuncture in the treatment of postprandial distress syndrome.

INPLASY registration number: This protocol was registered with the International Platform of Registered Systematic Review and Meta-Analysis Protocols (INPLASY) on 23 December 2021 and was last updated on 23 December 2021 (registration number INPLASY2021120101).

\section{INTRODUCTION}

Review question / Objective: Traditional Chinese medicine advocates using acupuncture for the treatment of postprandial distress syndrome (PDS) in people with functional dyspepsia (FD), but large clinical trials of acupuncture have produced controversial results. We perform this meta-analysis to evaluate the efficacy and safety of acupuncture in the treatment of postprandial distress syndrome. P: The subjects were patients diagnosed with 
postprandial discomfort syndrome. I: Patients received acupuncture as a treatment. C: Subjects received acupuncture in non-acupoint points, Gasmotin, moroxyline, or other reported interventions. 0 : The main outcomes included in this meta-analysis are the overall therapeutic rate, the Symptom Index of Dyspepsia (SID), the Hospital Anxiety Depression Scale (HADS), the Nepean Dyspepsia Index (NDI), and the incidence of the side effects.

Condition being studied: Efficacy and safety of acupuncture in the treatment of postprandial distress syndrome.

\section{METHODS}

Participant or population: The subjects were patients diagnosed with postprandial discomfortsyndrome.

Intervention: Patients received acupuncture as a treatment.

Comparator: Subjects received acupuncture in non-acupoint points, Gasmotin, moroxyline, or other reported interventions.

Study designs to be included: Clinical trials.

Eligibility criteria: 1 . The study must be a clinical randomized controlled trials; 2. Participants or outcomes and interventions should meet the inclusion criteria ; 3 . Enrollment of patients did not have any organic digestive diseases; 4 . Data must be complete and available ; 5. Any conference papers, reviews, case reports, experience summaries, and repeated literature will not be rejected.

Information sources: Only randomized controlled trials will be included from the following databases: China national knowledge infrastructure (CNKI), Medline, Cochrane Central, Web of Science, and Clinical Trial.

Main outcome(s): The main outcomes included in this meta-analysis are the overall therapeutic rate, the Symptom
Index of Dyspepsia (SID), the Hospital Anxiety Depression Scale (HADS), the Nepean Dyspepsia Index (NDI), and the incidence of the side effects.

Quality assessment / Risk of bias analysis: Assessment of risk of bias will be analyzed by Revman 5.4.1(RevMan; The Cochrane Collaboration, Oxford, UK) according to Cochrane Handbook for Systematic Reviews of Interventions in the following aspects: 1. Random sequence generation 2. Allocation concealment 3 . Blinding of participants and personnel 4. Blinding of outcome assessment 5 . Incomplete outcome data 6 . Selective reporting 7 . Other bias. A summary of the risk of bias was provided in Figure 2.

Strategy of data synthesis: Each outcome will be represented by using standardized mean difference (SMD) with a $95 \%$ confidence interval (Cl). STATA software (Stata Corporation, College Station, TX) was used to perform forest plot graphics and funnel plots. All statistical tests were bilateral significant levels: $\mathbf{P}=\mathbf{0 . 0 5}$. Heterogeneity of included trials will be calculated by Q-statistic and 12 test. We calculated the $Q$ statistic to evaluate heterogeneity, and $P \leq 0.01$ was considered to have a statistical significance of the $Q$ statistical test.

\section{Subgroup analysis: No subgroup analysis.}

Sensitivity analysis: Sensitivity analysis will be carried out if necessary to evaluate the stability of our result.

Country(ies) involved: China.

Keywords: postprandial distress syndrome, acupuncture, functional dyspepsia, gastroenterology

Contributions of each author:

Author 1 - Ganchen Xiao.

Email: xiaoganchen722@gzy.edu.cn

Author 2 - Yingtao Zhao.

Email: 2737960694@qq.com

Author 3 - Xingyu Chen.

Email: 1256242083@qq.com 